\title{
Characterization of Functionalized Polyurethane Foam for Lead Ion Removal from Water
}

\author{
Subhashini Gunashekar and Nidal Abu-Zahra \\ Materials Science and Engineering Department, University of Wisconsin-Milwaukee, 3200 N Cramer Street,
} Milwaukee, WI 53201, USA

Correspondence should be addressed to Subhashini Gunashekar; gunashe2@uwm.edu

Received 14 October 2013; Accepted 28 January 2014; Published 10 March 2014

Academic Editor: Harald W. Ade

Copyright (C) 2014 S. Gunashekar and N. Abu-Zahra. This is an open access article distributed under the Creative Commons Attribution License, which permits unrestricted use, distribution, and reproduction in any medium, provided the original work is properly cited.

Polyurethane foams functionalized with sulfonic acid groups are used in this study to exchange lead $\left(\mathrm{Pb}^{2+}\right)$ ions from aqueous solutions. Toluene-2, 4-diisocyanate, 2,6-diisocyanate (TDI) was reacted with Polypropylene glycol 1200 (PPG) in $2: 1$ molar ratio to form a linear prepolymer. The linear prepolymer was further polymerized using N,N-bis(2-hydroxyethyl)-2-aminoethanesulfonic acid (BES), which acts both as a chain extender and an ion-exchanger for $\mathrm{Pb}^{2+}$ ions. The functionalized polyurethane foam was characterized by Fourier transform infrared spectroscopy (FTIR), gel permeation chromatography (GPC), scanning electron microscopy (SEM), and energy dispersive X-ray spectroscopy (EDX). The $\mathrm{Pb}^{2+}$ ion exchange capacity was determined using an Inductively Coupled Plasma Mass Spectrometer (ICP-MS). The maximum $\mathrm{Pb}^{2+}$ ion exchange capacity of the foam was found to be $51 \mathrm{ppb} / \mathrm{g}$ from a $100 \mathrm{ppb} \mathrm{Pb}^{2+}$ solution over a period of two hours. In addition, $\mathrm{pH}$ analysis was carried out on the foam composition with the best $\mathrm{Pb}^{2+}$ ion removal capacity. The $\mathrm{pH}$ results based on two-hour exposures showed that the functionalized polyurethane foam performed better at lower $\mathrm{pH}$ levels.

\section{Introduction}

Polyurethane foams are considered to be one of the best commercially available insulation materials. They possess good thermal insulating properties, low moisture-vapor permeability, high resistance to water absorption, and a relatively high specific strength. Another advantage of polyurethane foam systems is that the synthesis can be tailored to various specific applications. The major components of polyurethane foams are an isocyanate and a polyol (or a mixture of polyols). A blowing agent and a catalyst are used to accelerate the foam formation. The foams can be synthesized as open-cell foams or as closed-cell foams based on the initial raw materials concentration. Recently, the cellular form of this polymer has intrigued researchers to explore other novel applications for this material.

Open cell polyurethane foams have been shown to exhibit a reasonable amount of ion exchange capacity and hence are being considered as suitable ion exchange media for heavy metal ions removal [1]. Braun and Farag [2] showed that open-pore polyurethane microspheres exhibit low cation exchange capacity. Their research on foams prepared by chemical bonding of specific functional groups (-SH) was used to adsorb mercury ions from mercury (II) chloride and methyl mercury (II) chloride in the range of 0.4 to $400 \mathrm{ppb}$. This also led researchers to consider the functionalization of polyurethane foams as another effective technique to eliminate heavy metal ions either by adsorption or preconcentration mechanisms. Functional groups like hydroxyl, ketone, and carboxylic acids have been found to adjust the surface energy and improve the hydrogen binding in polymers [3]. This property is the basis for functionalization of polyurethane foams either by surface or structural modification. Yeon et al. [4] developed a continuous electrodeionization medium to deionize aqueous media to a high level without the need for chemical regeneration using functionalized polyurethane foam. A chain extender was used during synthesis to functionalize the prepolymer. This led to the bulk functionalization of polyurethane foam with an increase in ion exchange capacity as the number of sulfonic acid groups increased. 
Functionalization of polyurethane foams by surface modification has led to systems capable of adsorbing heavy metal and trace metal ions. Surface modification of the foam usually involves the coupling of a sorbent to the polyurethane foam through an AZO group [5-8]. Fournier et al. [9] have used a copper catalyst to aid the cycloaddition of various azide compounds to modify the alkyne groups along the polyurethane backbone to obtain a side-chain functionalized cross linked polyurethane by means of click chemistry. Researches on graft and composite foam systems have established their efficiency to treat heavy metal ions [10-12]. Polyurethane foams modified by grafting acrylonitrile and acrylic acid by gamma irradiation method reported by Meligi [10] have shown that the adsorption of heavy metal ions like $\mathrm{Zn}$ (II), $\mathrm{Fe}(\mathrm{II}), \mathrm{Ca}(\mathrm{II}), \mathrm{Ni}(\mathrm{II}), \mathrm{Cu}(\mathrm{II})$, and $\mathrm{Pb}(\mathrm{II})$ was affected by $\mathrm{pH}$, atomic weight, and initial contaminant concentration.

In a study published by Jang et al. [11], hydroxyapatite(HAP-) polyurethane foams were synthesized for $\mathrm{Pb}^{2+}$ ion adsorption. A maximum adsorption capacity was determined to be $150 \mathrm{mg} / \mathrm{g}$ for the composite with $50 \mathrm{wt} \%$ HAP. The study also concluded that higher HAP concentration exhibited higher $\mathrm{Pb}^{2+}$ ion adsorption capacity. Less uniform dispersion of HAP in the foam led to slower adsorption and adsorption was dominant at higher $\mathrm{pH}$ levels. Another type of polyurethane composite foam containing alginate was synthesized by Sone et al. [12], which had a structure similar to a weak cation exchanger. Results from this study showed that $\mathrm{Pb}^{2+}$ adsorption was sensitive to lower $\mathrm{pH}$ and competing cations and the $\mathrm{Pb}^{2+}$ ion adsorption using this foam ranged from $20 \mathrm{ppb}$ to $\sim 100 \mathrm{ppb}$ in $2 \mathrm{hrs}$.

However, the research summary described above does not address bulk functionalization of polyurethane foams for selective elimination of heavy metal ions. To address this we focused our research work on the development of a bulk functionalized polyurethane foam system using a chain extender. Chain extenders are low-molecular multifunctional species. They can be used to balance the backbone structure of polymers [13]. This makes them suitable for selective elimination of heavy metal ions. In this paper, we discuss the modified synthesis process formulated by Yeon et al. [4] using $\mathrm{N}, \mathrm{N}$-bis(2-hydroxyethyl)-2-aminoethanesulfonic acid (BES) chain extender to eliminate $\mathrm{Pb}^{2+}$ ions from aqueous media. In addition, we will present a broad range of characterization of the functionalized foam in order to better understand the capabilities and limitations of the foam to remove $\mathrm{Pb}^{2+}$ ions from water.

\section{Experimental Work}

2.1. Materials. Polypropylene glycol 1200 (PPG; Sigma Aldrich Co. LLC) was dried in a vacuum oven at $70^{\circ} \mathrm{C}$ for 24 hours. Toluene diisocyanate (TDI; $2.4-80 \%$ and $2.6-20 \%$, Alfa Aesar), Dimethyl Sulfoxide (DMSO; Alfa Aesar, 99.9\% pure), N,N-bis(2-hydroxyethyl)-2-aminoethanesulfonic acid (BES; Alfa Aesar, 99\% pure), Dibutyltin dilaurate (DBTL, Sigma Aldrich, 95\% pure), Polysiloxane surfactant (Sigma Aldrich), and Nitrogen gas (Airgas, $\mathrm{O}_{2}$ free UHP) were used.
2.2. Synthesis of Functionalized Polyurethane Foam. The experimental set up consists of a 3-neck round bottom reaction flask placed in an oil bath fitted with a mechanical stirrer and a condenser at the center neck, nitrogen gas inlet and outlet at the right neck, and a drop funnel at the left neck. The reaction was carried out at $65-70^{\circ} \mathrm{C}$ in an inert atmosphere. The 3-neck flask was initially charged with TDI and allowed to stabilize at $70^{\circ} \mathrm{C}$ in a saturated nitrogen atmosphere. A drop funnel was filled with a preweighted amount of PPG which was added drop wise and allowed to react with TDI for 3-4 hours until an initial isocyanate content of $11-12 \%$ was reached as described in ASTM D5155 procedure.

A preweighted amount of BES dissolved in DMSO (amount of DMSO used was based on the solubility limit of $\mathrm{BES}$ in $\mathrm{DMSO}$ ) at $70^{\circ} \mathrm{C}$ was added drop wise into the reaction flask. The reaction was allowed to proceed until a final isocyanate content of $7-8 \%$ was reached. The tin catalyst was added at the end and the prepolymer was decanted into a glass mold. Based on the amount of PPG used, a preweighted amount of distilled water was added as a blowing agent along with the surfactant. The mixture was then mixed using a mechanical stirrer at $2500-3000 \mathrm{rpm}$ for $10-15$ seconds. This initiates the reaction of water with the remaining isocyanate groups forming an intermediate compound and eventually releasing $\mathrm{CO}_{2}$ gas to form the cellular structure [14].

In order to determine the optimum (1) chain extender (BES) content, (2) molar ratio of PPG and TDI, and (3) chain extender reaction time (CERT), a set of experiments were designed as discussed below. The objective of these experiments was to characterize the effect of these parameters on the physical properties and performance (i.e., ion exchange capacity) of the foam. Table 1 shows a detailed list of the compositions used to prepare the foam samples. Samples A1 to A4 were prepared with different BES/DMSO contents, while other parameters are maintained constant. Samples B5 to B7 were prepared with different PPG/TDI ratios, whereas samples $\mathrm{C} 8$ to $\mathrm{C} 10$ were prepared with different chain extender reaction times (CERT). Samples A3, B6, and C8 have the same composition, but they are used to study different design variables.

2.3. Fourier Transform Infrared (FTIR) Spectroscopy. The functionalized polyurethane foam samples were characterized by a BRUKER vector 22 FTIR with a DTGS (deuterated triglycine sulfate) detector and a PIKE 3-12 multibounce $\mathrm{Zn}$-Se variable angle ATR. The incidence angle was set to 80 during characterization. The foam samples were cut into strips of $3^{\prime \prime} \times 1^{\prime \prime} \times 0.5^{\prime \prime}$ and were washed in distilled water and dried in a vacuum oven before testing. The IR spectrum was measured from 400 to $4000 \mathrm{~cm}^{-1}$ to confirm the presence of sulfonic groups in the polymer structure.

2.4. Gel Permeation Chromatography (GPC). The polyurethane foam samples were analyzed using a Varian ProStar HPLC system using a PLgel $5 \mathrm{~mm}$ mixed C column. HPLC grade dimethyl formamide (DMF) was used as the eluent with a flow rate of $1 \mathrm{~mL} / \mathrm{min}$. The UV detector was set to detect sample at a wavelength of $569 \mathrm{~nm}$. Foam samples were dissolved in DMF to get a $0.5 \% \mathrm{w} / \mathrm{v}$ concentration and $25 \mu \mathrm{L}$ 
TABLE 1: Foam composition based on various processing variables.

\begin{tabular}{|c|c|c|c|c|c|c|c|c|c|}
\hline Variable & Sample & PPG (g) & TDI (g) & BES (g) & DMSO $(\mathrm{g})$ & DBTL (g) & Surfactant (g) & Water (g) & CERT (min) \\
\hline \multirow{4}{*}{ BES/DMSO } & $\mathrm{A} 1$ & \multirow{4}{*}{50} & \multirow{4}{*}{18.3} & 0 & 0 & \multirow{4}{*}{2} & \multirow{4}{*}{0.25} & \multirow{4}{*}{6} & \multirow{4}{*}{40} \\
\hline & $\mathrm{A} 2$ & & & 3.4 & 7.8 & & & & \\
\hline & $\mathrm{A} 3$ & & & 6.7 & 15.7 & & & & \\
\hline & A4 & & & 10.1 & 23.5 & & & & \\
\hline \multirow{3}{*}{ PPG/TDI } & B5 & \multirow{3}{*}{50} & 9.1 & & \multirow{3}{*}{15.7} & \multirow{3}{*}{2} & \multirow{3}{*}{0.25} & \multirow{3}{*}{6} & \multirow{3}{*}{40} \\
\hline & B6 & & 18.3 & 6.7 & & & & & \\
\hline & B7 & & 27.4 & & & & & & \\
\hline \multirow{3}{*}{ CERT } & C9 & \multirow{3}{*}{50} & \multirow{3}{*}{18.3} & & \multirow{3}{*}{15.7} & \multirow{3}{*}{2} & \multirow{3}{*}{0.25} & \multirow{3}{*}{6} & 60 \\
\hline & $\mathrm{C} 8$ & & & 6.7 & & & & & 40 \\
\hline & $\mathrm{C} 10$ & & & & & & & & 90 \\
\hline
\end{tabular}

of the sample was injected into the column [15]. The main purpose of this analysis was to determine the effect of the chain extender (BES) and the solvent (DMSO) on the foam structure during synthesis.

2.5. Sample Preparation for ICP-MS Analysis. An Inductively Coupled Plasma Mass Spectrometer (ICP-MS) was used for trace metal analysis at ppb levels. The demolded functionalized foam was cut into one gram cubes, maintaining a constant volume. Polyurethane foam requires homogeneous rearrangement of its polymeric bonds for effective ion exchange, as the foam does not experience stress-free conditions after synthesis [4]. The foam cubes were washed in distilled water and conditioned in $2 \mathrm{~N} \mathrm{NaCl}$ solution for four hours to ensure rearrangement of the polymeric bonds.

Standard $\mathrm{Pb}^{2+}$ solutions of $100 \mathrm{ppb}$ were prepared from a stock solution of 1 part per million ( $\mathrm{ppm}$ ). The conditioned foams were soaked in $25 \mathrm{~mL}$ of $\mathrm{Pb}^{2+}$ solution in a beaker with a magnet. The beaker was then covered to avoid contamination and placed on a stirrer. Foam cubes were submersed in the $\mathrm{Pb}^{2+}$ solution for various time periods. $10 \mathrm{~mL}$ of each sample was filtered into $15 \mathrm{~mL}$ centrifuge tubes. Nitric acid was added to stabilize the $\mathrm{Pb}^{2+}$ ions in the sample during storage and for ICP-MS analysis.

\section{Results and Discussion}

The study samples were prepared according to Table 1, shown earlier. The physical characteristics of the foam, such as its color, odor, pore size, and structure, depend primarily on the DMSO (solvent) content. Foam samples A1, A2, and A3, which were prepared with lower amounts of DMSO and lower CERTs, had a similar pale yellow color and were odorless. Foam samples which were prepared with higher amounts of DMSO and/or CERTs were much darker (brownish) and had a strong odor. Similarly, the flexibility of the foam seemed to increase with increasing DMSO content and CERT as was observed with samples A3, A4, C9, and C10. This may be due to the cleavage of the polymeric bonds connecting the hard segments in the foam formed by DMSO, since it is a strong organic solvent capable of dissolving the polyurethane foam on prolonged exposures. On the other hand, the rigidity of the foam increased with increasing TDI content (e.g., B7) due to the excess hard segments formed by the quasiprepolymer in the foam [16]. Lower amounts of TDI in sample B5 did not favor foam formation leading us to the conclusion that the ratio of soft and hard segments (polyol: isocyanate) should be higher than $1: 1$ to form an isocyanate terminated true prepolymer [16].

3.1. Fourier Transform Infrared (FTIR) Spectroscopy. The FTIR spectrum of the foam samples A1 to A4 with varying BES/DMSO content is shown in Figure 1. Sample A1 shows a clear C-O-C peak at $1100 \mathrm{~cm}^{-1}$ as it does not contain any chain extenders with the sulfonic groups. The O-S-O peaks start to appear at $\sim 1060 \mathrm{~cm}^{-1}$ in other samples and this peak partially overlaps with the $\mathrm{C}-\mathrm{O}-\mathrm{C}$ band. The asymmetrically stretched $\mathrm{S}=\mathrm{O}$ peak appears at $1350 \mathrm{~cm}^{-1}$. The urethane carbonyl peaks appear at $1700 \mathrm{~cm}^{-1}$. The $\mathrm{NH}$ band and $\mathrm{H}-\mathrm{NH}$ band appear at $3000 \mathrm{~cm}^{-1}$ [4]. These indicate that the sulfonic acid functional groups were successfully integrated into the polyurethane backbone. The $-\mathrm{CH}$ stretching vibrations from the polyol appear at $2850-3000 \mathrm{~cm}^{-1}$ followed by the $-\mathrm{OH}$ and $-\mathrm{NH}$ stretching that can be seen at $3200-3300 \mathrm{~cm}^{-1}$ [17]. The $-\mathrm{CH}$ bending vibrations due to substitution groups in alkenes and aromatics are usually seen between 850$950 \mathrm{~cm}^{-1}$ [18]. The gradual appearance of a peak in this region as the BES content and CERT is increased confirms the incorporation of the BES chain extender into the polymer backbone.

Sung and Schneider [19] have reported that the urea microdomains in polyurethane foams are known to possess bidentate hydrogen bonding which is associated with a $1640 \mathrm{~cm}^{-1}$ absorbance observed via FTIR. The presence of hydrogen bonding also leads to the hard segments packing in an organized manner. In another study, Aneja and Wilkes [20] compared the isomers of TDI and determined that the $1640 \mathrm{~cm}^{-1}$ absorbance was found to decrease as the 2,4 TDI isomer content increased and suggested that the symmetry of the isocyanate moiety had a considerable impact on the packing behavior of the urea hard segments. From Figure 2, it is clearly evident that samples $\mathrm{A} 3(\mathrm{~B} 6 / \mathrm{C} 8)$ and B7 do not show any significant peaks at $1640 \mathrm{~cm}^{-1}$. This may be due to the foam composition since the samples differ only by an excess of 0.5 moles of TDI in each case, which may not be significant 


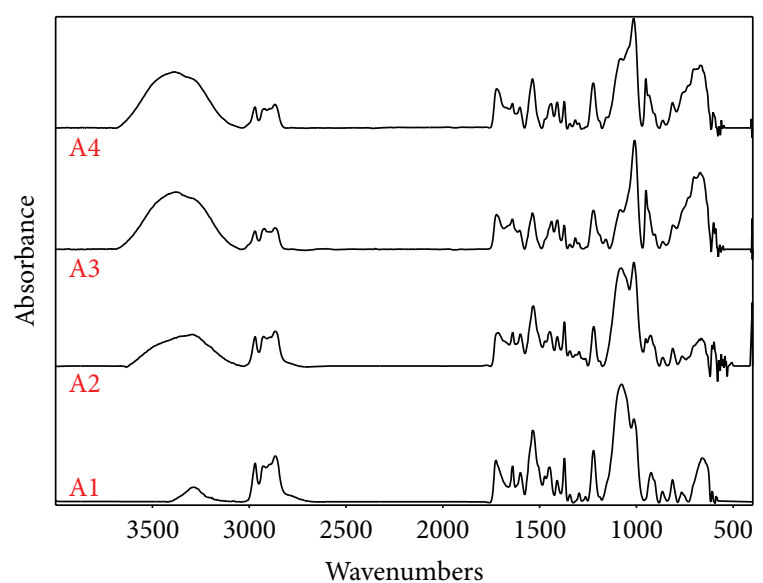

Figure 1: FTIR spectrum of samples A1-A4.

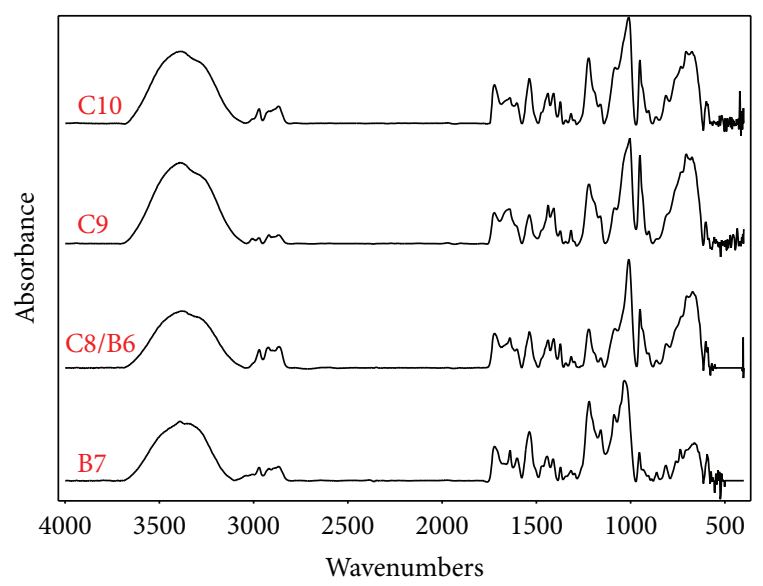

FIGURE 2: FTIR spectrum of samples B7-C10.

to show any changes in absorbance. The effect of CERT can be observed in the absorbance spectrums for samples A3, C9, and $\mathrm{C} 10$. The $\mathrm{C}-\mathrm{O}-\mathrm{C}$ and $\mathrm{O}-\mathrm{S}-\mathrm{O}$ peaks start to overlap at $1100 \mathrm{~cm}^{-1}$ as the CERT increases; they seem to merge to form a single peak.

3.2. Gel Permeation Chromatography (GPC). BES is a chain extender which links the isocyanate groups in the linear polymer chains through its hydroxyl groups, thereby increasing the molecular weight of polyurethane. DMSO is a strong organic solvent that dissolves polyurethane as well as BES. Since BES is insoluble in other organic solvents, the use of DMSO cannot be eliminated from the foam synthesis process. Hence, both BES and DMSO have an opposite effect on the molecular weight of the resulting polyurethane system.

GPC analysis shows that the retention time needed to elute higher molecular weight or larger molecules decreases as the DMSO content and CERT increase during synthesis. This can be seen in Figure 3 for samples A1-A4. Sample A1 has no DMSO and it elutes at 11.4 minutes. As the amount of DMSO increases in samples A2-A4, we can see larger molecules eluting earlier than 11.4 minutes followed by
TABLE 2: Retention times of foam samples.

\begin{tabular}{lclccc}
\hline Sample & \multicolumn{5}{c}{ Peak retention time (mins) } \\
\hline A1 & 11.4 & & & & \\
A2 & 9.5 & 11.3 & & & \\
A3 & 8.6 & 11.1 & 11.6 & 13.7 & 14.6 \\
A4 & 7.7 & 11.0 & 11.7 & & \\
B7 & 9.2 & 11.4 & & 13.9 & \\
C9 & 9.4 & 11.0 & 11.4 & 13.9 \\
C10 & 9.4 & 9.7 & 10.6 & 11.7 & 15.3 \\
\hline
\end{tabular}
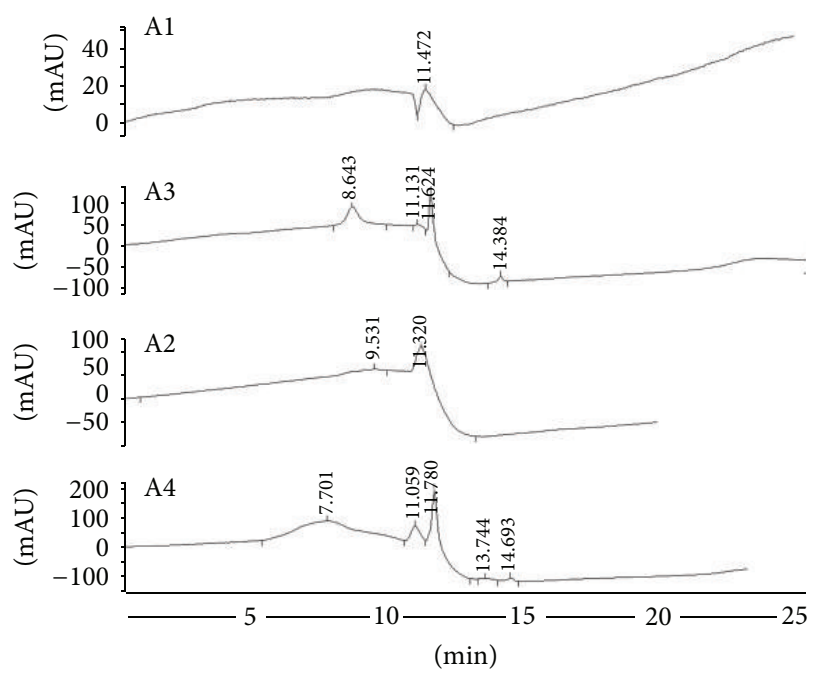

FIGURE 3: High and low molecular weight compounds of functionalized polyurethane foam samples based on BES/DMSO content.

smaller molecules after 11.4 minutes. This can be attributed to the cleavage of polyurethane chains forming in samples with higher DMSO content. The peak retention times for all the tested samples are summarized in Table 2.

Samples A2 and B7 seem to show a similar elution trend. A2 has a lower amount of DMSO and B7 has a higher TDI content in its composition. This provides additional isocyanate groups for functionalization, therefore lowering the ability of DMSO to break the polymer chains during synthesis. Hence, increasing the TDI content in the foam composition without affecting the structural integrity of the foam may be a way to reduce the cleaving effect of DMSO.

Similarly, Figure 4 shows GPC analysis for samples B7C10. It is evident that increasing CERT has the same effect in samples C9 and C10. Larger molecules start to elute after 9.4 minutes and, with reasonable amounts of DMSO, higher CERT seems to cleave polymer chains to a greater extent resulting in a large molecular weight distribution in the foam sample. This is confirmed by the higher number of molecular segments that are seen to elute at 9.4, 9.7, 10.6, 11.7, and 15.3 minutes, respectively, in sample $\mathrm{C} 10$ which was prepared with the highest CERT. These factors influence the distribution of the functional group in the foam and affect the $\mathrm{Pb}^{2+}$ ion removal capacity of the foam. 

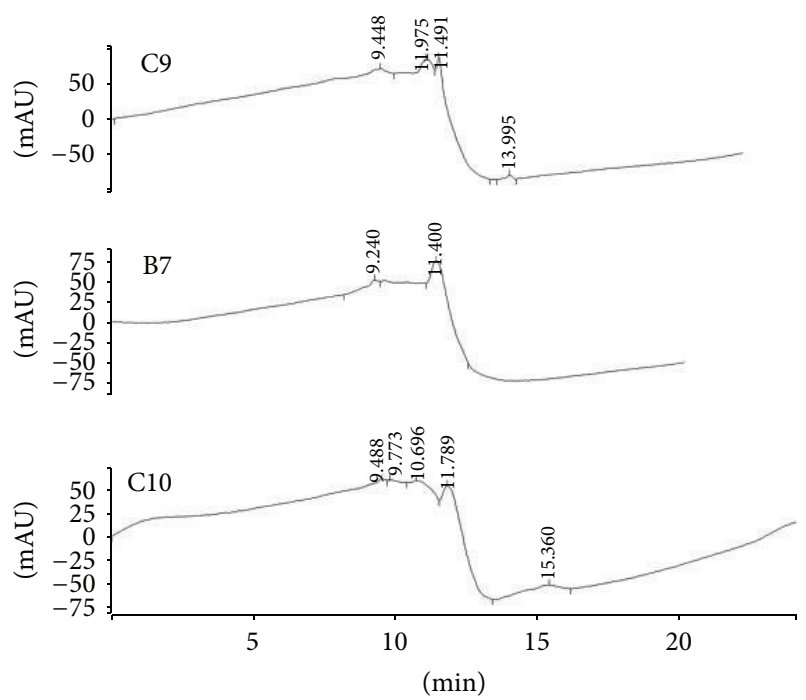

FIgURE 4: High and low molecular weight compounds of functionalized polyurethane foam.

3.3. Scanning Electron Microscopy and Energy Dispersive $X$-Ray Analysis (SEM/EDX). Foam samples were analyzed using a Topcon SM-300 SEM/EDX instrument. The samples were coated using a sputter coater before analysis. Figure 5 confirms the open cell structure of an unconditioned foam sample with A3 composition. Elemental analysis by EDX performed on the foam sample, after soaking in $100 \mathrm{ppb}$ standard $\mathrm{Pb}^{2+}$ solution for 2 hours, shows the presence of $\mathrm{Pb}^{2+}$ in the bulk of the foam sample along with sulfur and various other elements, as shown in Figure 6. This confirms the $\mathrm{Pb}^{2+}$ ion exchange with sulfonic acid groups in the functionalized polyurethane foam.

3.4. Inductively Coupled Plasma-Mass Spectrometry (ICP$M S)$. ICP-MS is a powerful analytical technique that allows detection of trace elements at parts per billion and parts per trillion levels. Lead ion exchange capacity of the synthesized foam was measured using ICP-MS.

The synthesized foam samples were cut into symmetrical cubes, weighing about 1 gram, and were conditioned in $2 \mathrm{~N}$ $\mathrm{NaCl}$ solution for 4 hours before soaking in a $100 \mathrm{ppb}$ lead solution for a period of 2 hours. The lead solution was filtered and analyzed by ICP-MS to determine the final lead concentration in the solution. The experiments were repeated with two grams of foam samples. The results of this analysis are shown in Figure 7.

Foam samples $\mathrm{A} 1$ (0 moles $\mathrm{BES})$ showed some $\mathrm{Pb}^{2+}$ removal in the absence of sulfonic functional groups, which confirms that the mechanism of lead ion removal is not only by ion exchange but may also be due to surface adhesion or absorption [21]. The lead removal capacity steadily increases as the chain extender (BES) content increases in samples A2 (0.02 moles BES) and A3 (0.03 moles BES). However, sample $\mathrm{A} 4$ (0.05 moles BES) exhibited lower $\mathrm{Pb}^{2+}$ removal. This may be a result of uneven distribution of functional groups in the bulk of the foam due to the disintegration of polymer

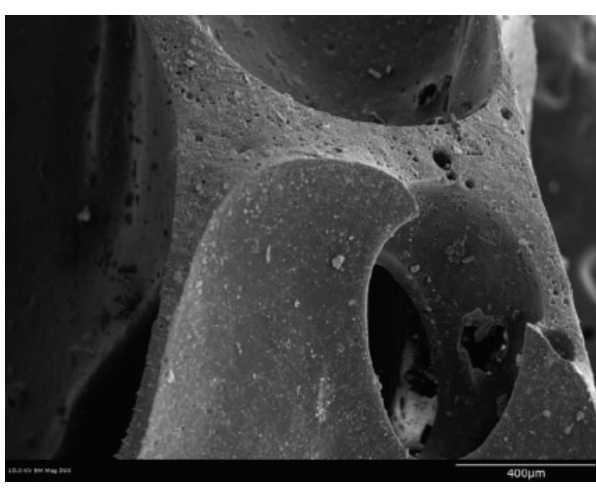

FIGURE 5: SEM image of polyurethane foam at 50x magnification.

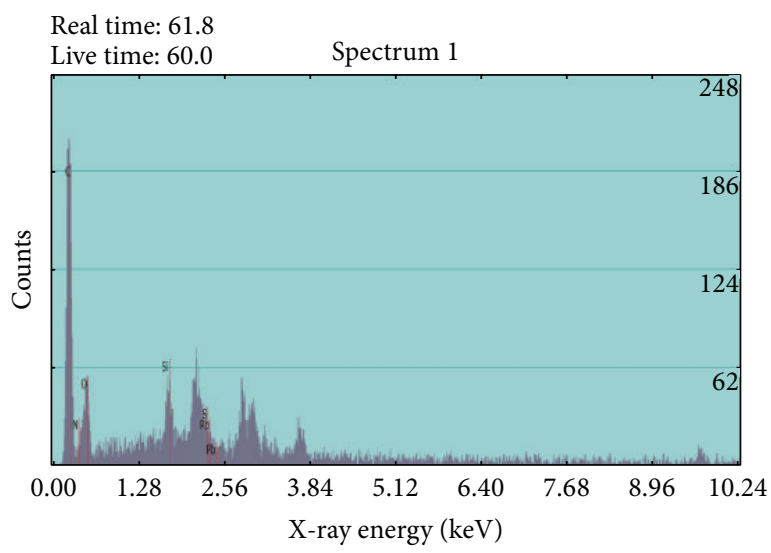

Figure 6: EDX elemental analysis.

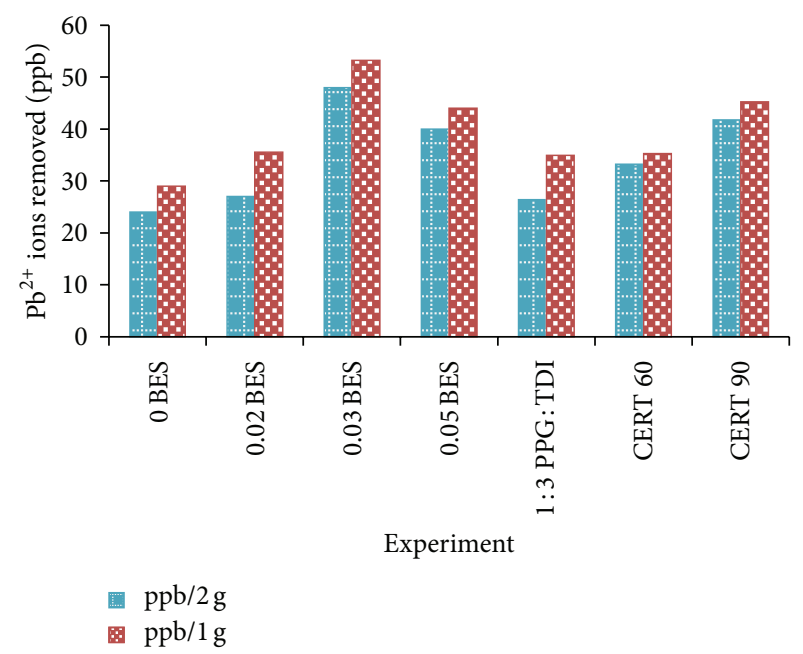

FIgURE 7: Lead removal capacity based on foam weight.

chains by excess DMSO used to dissolve higher amounts of BES. This is supported by a similar behavior observed with increasing the CERT in experiment C10. Foam samples with composition A3 exhibited the best lead removal capacity.

An increase in lead removal capacity for all the foam samples was observed when 2 grams of the foam was soaked 


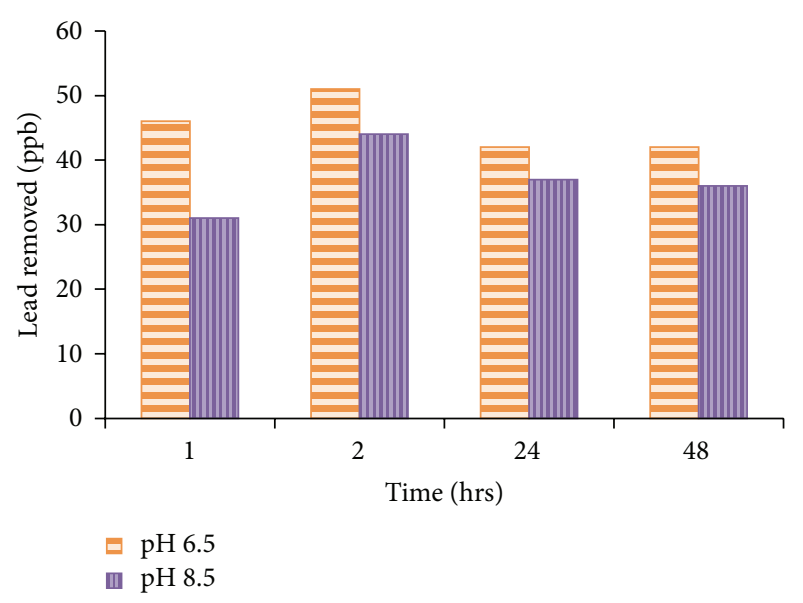

FIGURE 8: $\mathrm{pH}$ analysis of functionalized polyurethane foam.

in $\mathrm{Pb}^{2+}$ solution. This is clearly due to the availability of additional functional groups for ion exchange which increased the $\mathrm{Pb}^{2+}$ ion removal capacity from $48 \mathrm{ppb} / \mathrm{g}$ to $53 \mathrm{ppb} / 2 \mathrm{~g}$ of $\mathrm{A} 3$ foam.

3.5. pH Analysis. Based on the above results, A3 foam samples were analyzed at 6.5 and $8.5 \mathrm{pH}$ levels over a period of 1 to 48 hours, as shown in Figure 8. Since the foam is considered for drinking water applications, it was not tested at higher or lower $\mathrm{pH}$ levels. The lead removal capacity of the foam seemed to be higher at a $\mathrm{pH}$ of 6.5 , with a maximum lead removal capacity of $51 \mathrm{ppb} / \mathrm{g}$, after soaking the foam in lead solution for $2 \mathrm{hrs}$.

The lead removal capacity of the foam seemed to taper down and saturate after $2 \mathrm{hrs}$. Lead removal capacity of the foam also seemed to decrease as the $\mathrm{pH}$ of the solution increases to 8.5. This may be due to the neutralization of the sulfonic acid groups at higher $\mathrm{pH}$ levels which in turn diminishes the availability of functional groups for ion exchange [21]. The foam seems to have a better lead removal capacity at lower $\mathrm{pH}$ values for the same reason.

\section{Summary and Conclusions}

Polyurethane foam made of PPG and TDI segments with sulfonic functional groups was synthesized using BES chain extender dissolved in DMSO for $\mathrm{Pb}^{2+}$ ion removal from aqueous solutions. Various parameters, such as PPG/TDI ratio, BES/DMSO content, and CERT, were studied by designing a set of ten experiments. The outcome of each experiment (i.e., combination) was recorded to analyze the physical characteristics of the functionalized foam. The foams were conditioned in $2 \mathrm{~N} \mathrm{NaCl}$ solution to improve the ion exchange capacity and to realign the polymeric bonds.

FTIR characterization of the foam samples confirmed the presence of sulfonic groups in the polyurethane backbone. GPC characterization confirmed polymer chain disintegration due to higher DMSO content and increased CERT. This was further confirmed by ICP-MS analysis showing the lead removal capacity of the foams. Foam samples containing higher DMSO exhibit lower $\mathrm{Pb}^{2+}$ removal rate since the functional groups were not available uniformly throughout the bulk of the foam. EDX analysis confirmed the presence of $\mathrm{Pb}^{2+}$ in the foam samples soaked in a standard lead solution. Foam samples synthesized with $1: 2$ mole ratio of PPG : TDI and 0.6: 4 moles of BES : DMSO (based on moles of TDI) exhibited the best performance of lead removal. The measured lead removal capacity of the foam was $48 \mathrm{ppb} / \mathrm{g}$ and $51 \mathrm{ppb} / 2 \mathrm{~g} . \mathrm{pH}$ analysis of the foam showed reduction in its lead removal capacity at higher $\mathrm{pH}$ levels due to neutralization of sulfonic acid groups which lowered the number of functional sites available for ion exchange.

\section{Conflict of Interests}

The authors declare that there is no conflict of interests regarding the publication of this paper.

\section{Acknowledgments}

The authors would like to thank the members of Water Equipment Policies (WEP) and Industry-University cooperative research program (I/UCRC) for their support through the course of this research work. The authors would also like to extend their gratitude to Susan Krezoski from the Department of Chemistry and Biochemistry, UW-Milwaukee, for scheduling and coordinating the use of ICP-MS, and Dr. Xiangyang Liu and Dr. Marianna Orlova of the UWMilwaukee, biotech facility, for training and coordinating the use of GPC.

\section{References}

[1] R. L. MacBrayer D C Wysocki, Polyurethane Foams Formulation and Manufacture, Program Division, Technomic Publishing Company, Incorporated, 347 pages, 1998.

[2] T. Braun and A. B. Farag, "Polyurethane foams and microspheres in analytical chemistry. Improved liquid-solid, gas-solid and liquid-liquid contact via a new geometry of the solid phase," Analytica Chimica Acta, vol. 99, no. 1, pp. 1-36, 1978.

[3] J. G. A. Terlingen, "Introduction of functional groups at polymer surfaces by Glow discharge techniques," Tech Paper, Europlasma, 1993.

[4] K. H. Yeon, J. W. Lee, J. S. Lee, and S. H. Moon, "Preparation and characterization of cation-exchange media based on flexible polyurethane foams," Journal of Applied Polymer Science, vol. 86, no. 7, pp. 1773-1781, 2002.

[5] V. A. Lemos, L. N. Santos, A. P. O. Alves, and G. T. David, "Chromotropic acid-functionalized polyurethane foam: a new sorbent for on-line preconcentration and determination of cobalt and nickel in lettuce samples," Journal of Separation Science, vol. 29, no. 9, pp. 1197-1204, 2006.

[6] R. J. Krupadam, M. S. Khan, and S. Das, "Adsorption of fluoride from water by surface-functionalized polyurethane foam," Water Science and Technology, vol. 62, no. 4, pp. 759-765, 2010.

[7] E. A. Moawed, M. A. A. Zaid, and M. F. El-Shahat, "Analytical application of polyurethane foam functionalized with quinolin8-ol for preconcentration and determination of trace metal ions in wastewater," Journal of Analytical Chemistry, vol. 61, no. 5, pp. 458-464, 2006. 
[8] E. A. Moawed, M. A. Zaid, and M. F. El-Shahat, "Preparation, characterization and applications of polyurethane foam functionalized with resorcinol for quantitative separation and determination of silver(I) and mercury(II) from tap and wastewater," International Journal of Environmental Analytical Chemistry, vol. 84, no. 12, pp. 935-946, 2004.

[9] D. Fournier, B. G. de Geest, and F. E. du Prez, "On-demand click functionalization of polyurethane films and foams," Polymer, vol. 50, no. 23, pp. 5362-5367, 2009.

[10] G. A. Meligi, "Removal of some heavy metal ions using grafted polyurethane foam," Polymer, vol. 47, no. 1, pp. 106-113, 2008.

[11] S. H. Jang, B. G. Min, Y. G. Jeong, W. S. Lyoo, and S. C. Lee, "Removal of lead ions in aqueous solution by hydroxyapatite/ polyurethane composite foams," Journal of Hazardous Materials, vol. 152, no. 3, pp. 1285-1292, 2008.

[12] H. Sone, B. Fugetsu, and S. Tanaka, "Selective elimination of lead(II) ions by alginate/polyurethane composite foams," Journal of Hazardous Materials, vol. 162, no. 1, pp. 423-429, 2009.

[13] "Understanding Chain Extenders and Crosslinkers," SpecialChem, 2004, http://www.specialchem4polymers.com/ resources/articles/article.aspx?id=1777.

[14] M. Szycher, Szycher'S Handbook of PolyurethaneS, CRC Press, New York, NY, USA, 1999.

[15] G. Saunders and B. MacCreath, Application Note, Materials Testing and Research, Polymers, 2013, http://www.chem.agilent .com/Library/brochures/5990-7994-GPCorganics-Apr11-9lo .pdf.

[16] M. Chanda and S. K. Roy, Plastics Technology Handbook, chapter 2, CRC Press, New York, NY, USA, 3rs edition, 1998.

[17] J. Hansen, Characteristic IR Absorption Frequencies of Organic Functional Groups, 2006, http://www2.ups.edu/faculty/hanson/Spectroscopy/IR/IRfrequencies.html.

[18] P. Beauchamp, Spectroscopy Data Tables, 2010, http://www .csupomona.edu/ psbeauchamp/pdf/424_spectra_tables.pdf.

[19] C. S. P. Sung and N. S. Schneider, "Infrared studies of hydrogen bonding in toluene diisocyanate based polyurethanes," Macromolecules, vol. 8, no. 1, pp. 68-73, 1975.

[20] A. Aneja and G. L. Wilkes, "On the issue of urea phase connectivity in formulations based on molded flexible polyurethane foams," Journal of Applied Polymer Science, vol. 85, no. 14, pp. 2956-2967, 2002.

[21] C. E. Harland, Ion Exchange: Theory and Practice, Royal Society of Chemistry, 2nd edition, 1994. 

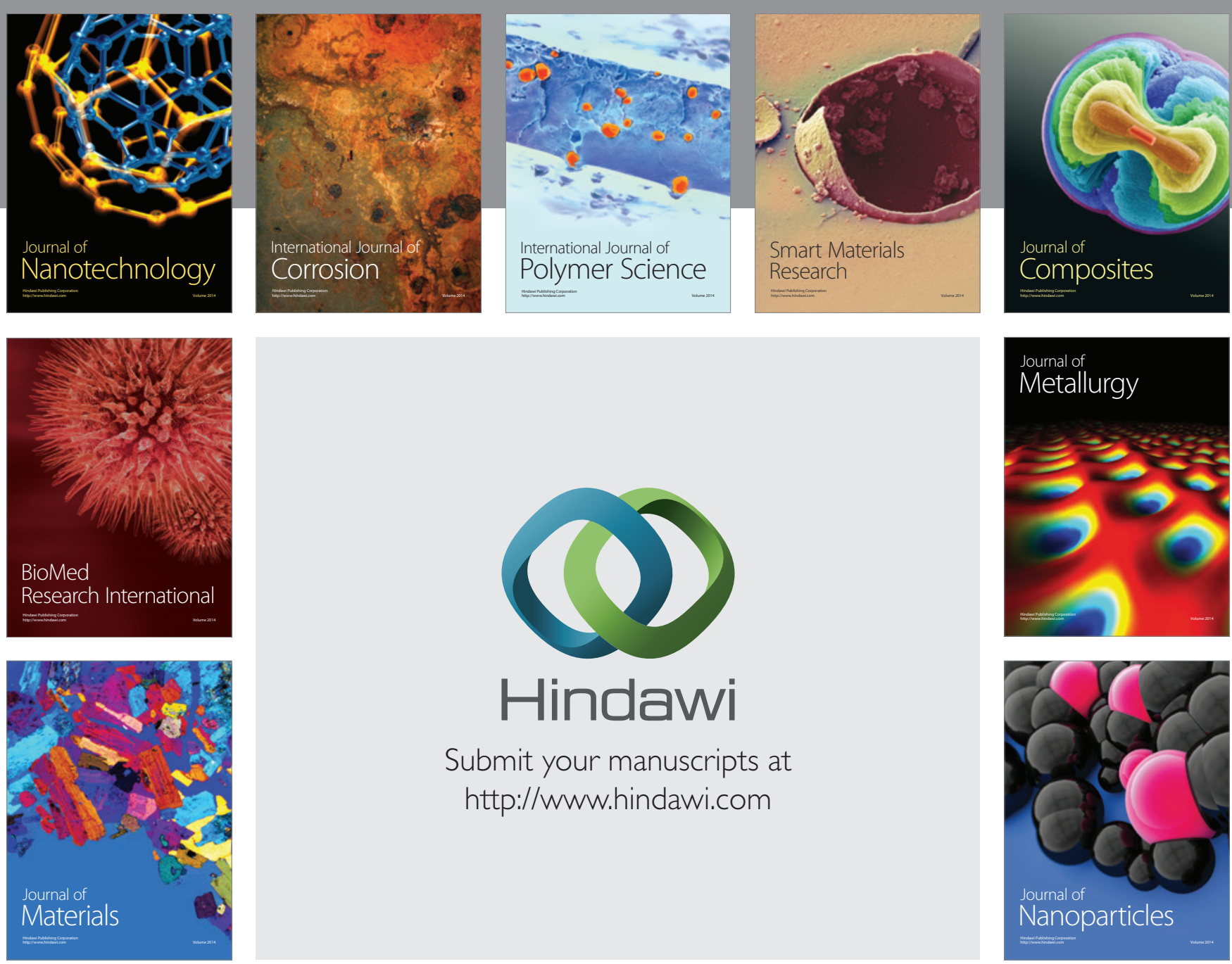

Submit your manuscripts at http://www.hindawi.com
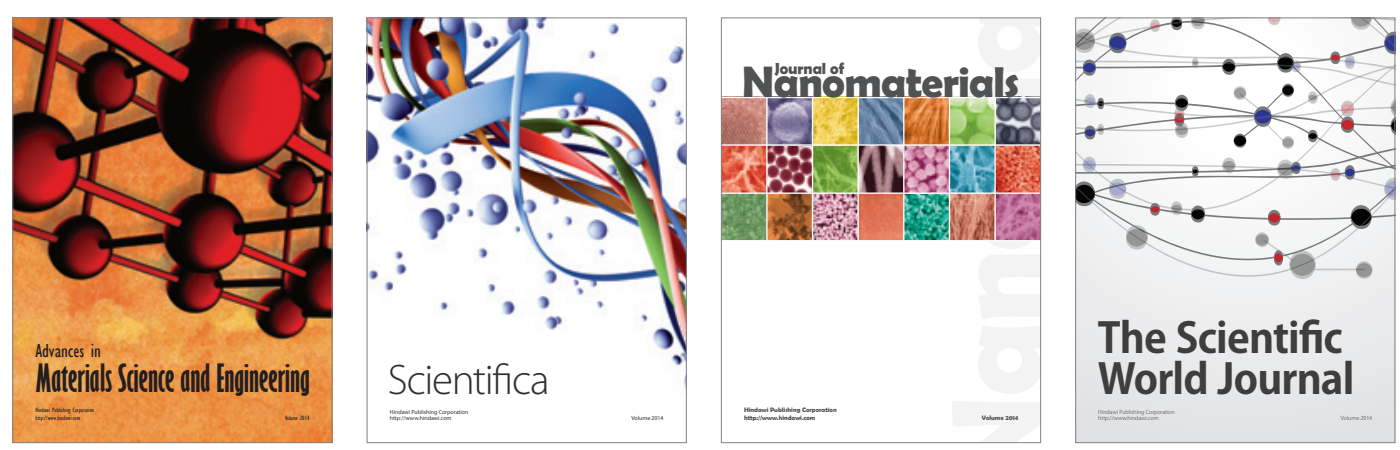

\section{The Scientific World Journal}
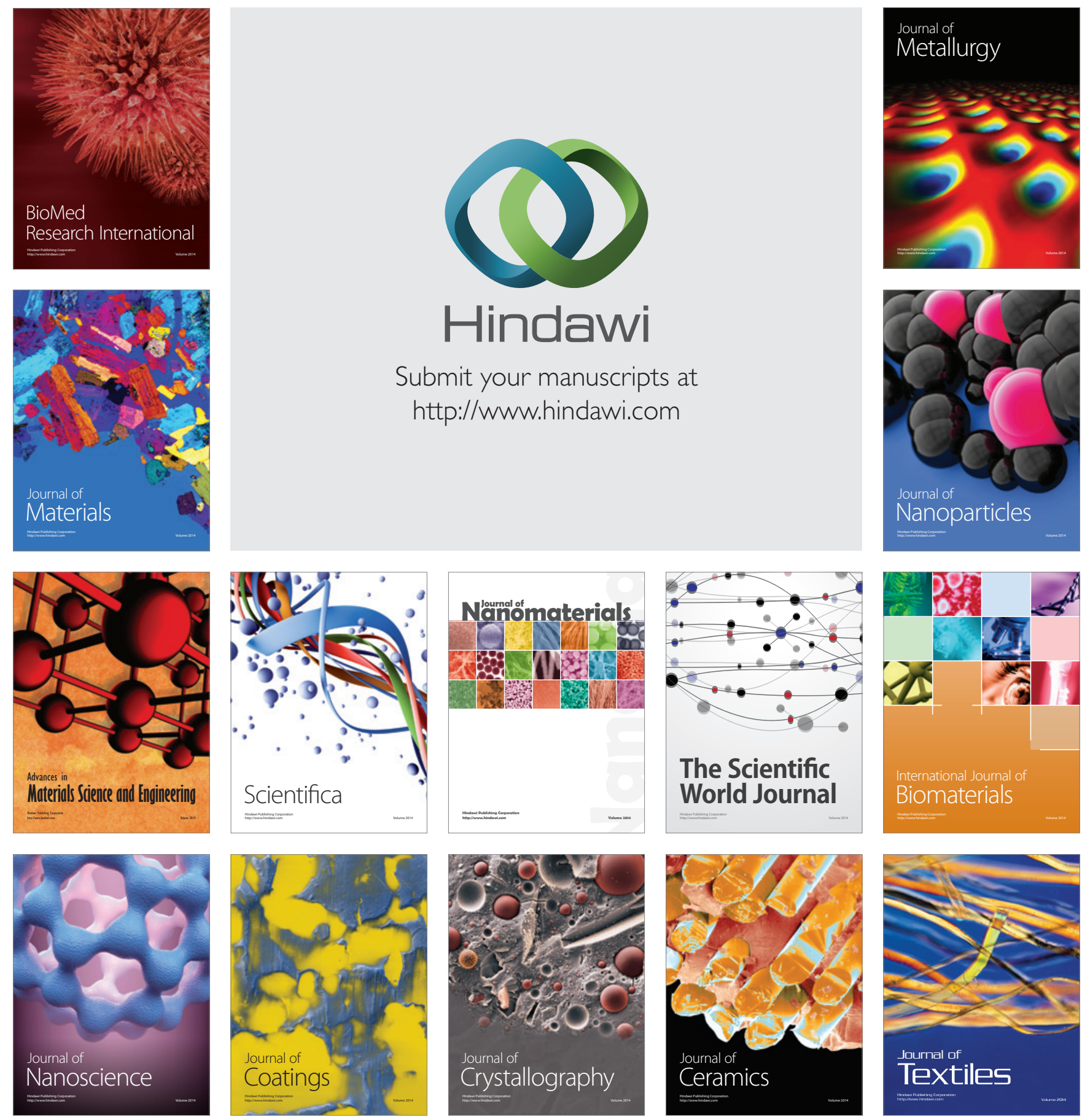